Research Article

\title{
Finite Element Analysis on Acoustic and Mechanical Performance of Flexible Perforated Honeycomb-Corrugation Hybrid Sandwich Panel
}

\author{
Jiaming Hu, ${ }^{1}$ Junyi Wang, ${ }^{1}$ Yu Xie, ${ }^{1}$ Chenzhi Shi, ${ }^{2}$ and Yun Chen $\mathbb{D}^{1}$ \\ ${ }^{1}$ State Key Laboratory of ASIC and System, Department of Microelectronics, Fudan University, Shanghai 200433, China \\ ${ }^{2}$ George W. Woodruff School of Mechanical Engineering, Georgia Institute of Technology, Atlanta, GA 30332, USA
}

Correspondence should be addressed to Yun Chen; chenyun@fudan.edu.cn

Received 5 March 2021; Accepted 6 May 2021; Published 17 May 2021

Academic Editor: Francisco Beltran-Carbajal

Copyright (c) 2021 Jiaming Hu et al. This is an open access article distributed under the Creative Commons Attribution License, which permits unrestricted use, distribution, and reproduction in any medium, provided the original work is properly cited.

\begin{abstract}
Since proposed, the perforated honeycomb-corrugation sandwich panel has attracted a lot of attention due to its superior broadband sound absorption at low frequencies and excellent mechanical stiffness/strength. However, most existing studies have assumed a structure made of high-strength materials and studied its performance based on the ideal rigid-wall model with little consideration for acoustic-structure interaction, thereby neglecting the structural vibrations caused by the material's elasticity. In this paper, we developed a more realistic model considering the solid structural dynamics using the finite element method (FEM) and by applying aluminum and rubber as the structural material. The enhancement of the low-frequency performance and inhibition of broadband absorption coexisted in low-strength rubbers, implying a compromise in the selection of Young's modulus to balance these two influences. Further analysis on thermal-viscous dissipation, mechanical energy, and average structural stress indicated that the structure should work right below the resonant frequency for optimization. Based on these findings, we designed a novel aluminum-rubber composite structure possessing enhanced low-frequency absorption, high resistance to shear load, normal compression, and thermal expansion. Our research is expected to shed some light on noise control and the design of multifunctional acoustic metamaterials.
\end{abstract}

\section{Introduction}

The vibration and noise caused by the environment are ubiquitous phenomena. Hazards caused by vibration and noise are versatile; strong vibration and noise affect a material's functional performance, reduce its service life, cause defects, and even destroy the inherent structure and fundamental properties. There are generally three noise control methods: source suppression, transmission path blocking, and receiver protection [1]. The most used and practical method is to block the propagation path by selecting a suitable acoustic material for noise absorption [2]. Soundabsorbing materials conventionally used primarily include porous and lost materials [3-5]. Microperforated panels (MPPs) initially proposed by Maa are also widely used structures that can absorb sound without the use of fibrous or porous materials $[6,7]$. MPPs can be made of metal, plastic, plywood, acrylic glass, and sheet materials and have a wide range of application $[8,9]$. However, these materials and structures are generally required to be at the wavelength scale, resulting in their large thickness with enormous weight, thereby limiting their range of application to high frequencies.

In low-frequency bands, resonator-based sound absorbers, such as membrane and Helmholtz absorbers, have been studied [10]. In the past decade, the introduction of acoustic metamaterials has introduced new methods of wave manipulation. Acoustic metamaterials consist of specially designed deep-subwavelength artificial acoustic structures and have plenty of extraordinary characteristics unprecedented in natural materials [11-13]. Some of these artificial composite materials achieve near-perfect sound absorption 
at low frequencies, breaking the mass density law limiting traditional sound-absorbing materials and accomplishing efficient sound absorption under lightweight conditions. However, most acoustic metamaterials still suffer from flaws, such as narrow working frequency bands and high manufacturing costs.

Since the invention of the honeycomb structure, it has attracted attention from numerous research fields because it is characterized by high stiffness [14], fatigue resistance, heat insulation [15], and noise reduction [16]. Honeycomb Sandwich panels have been widely used in the shipbuilding, transportation, aeronautics, and building industries [17]. To improve the absorption properties, combinations of MPPs and honeycomb Sandwich panels with a perforated top face sheet have become a popular solution for forming a Helmholtz resonator for sound absorption [18]. Tang et al. created a perforated honeycomb-corrugation hybrid (PHCH) Sandwich panel, which gained perfect sound absorption at low frequencies with super broadband [19]. Such a structure was proposed as a novel acoustic metamaterial and as a lightweight load-bearing structure [19]. The honeycomb-corrugation hybrid structure has also been found to be a high-performance, lightweight core. Combining a honeycomb structure of relative rigidness with a relatively soft corrugation can enhance specific compressive strength and energy absorption [20].

The effects of the vibration of the materials on their acoustic characteristics cannot be ignored, especially when they are designed to be lighter. Studies on these vibrational effects on a material's acoustic and mechanical properties have attracted more and more attention. In practice, the introduction of structural vibration excited by acoustic fields through a flexible membrane or board has been commonly used to design absorbers. Lee et al. studied the sound absorption of a rectangular enclosed acoustic cavity and a single-sided flexible microperforated plate and found that the vibration of the plate induced sound absorption peaks that could broaden the sound absorption bandwidth [21]. For the Sandwich panel, Sanada et al. added a flexible plate in the middle of the cavity with a single core of the honeycomb Sandwich structure perforated on the upper surface to create a Helmholtz resonant cavity. They then proposed a practical method to expand the effective sound absorption frequency range [22]. Physically, the flexible structure can contribute a greater degree of freedom to the system, which could potentially generate new absorption peaks.

In this paper, we developed our flexible perforated honeycomb-corrugation hybrid structure based on the model proposed by Tang et al. [23]. The wall and plate were modified to be flexible and have vibration and deformation. For the nonlinear dynamic analyses of complex mechanical components, it is necessary to apply an efficient modeling framework to reduce computational burden [24], so finite element analysis was conducted using COMSOL Multiphysics 5.4. Aluminum alloys and rubbers with different moduli were simulated, and their acoustic performances were evaluated by the sound absorption spectrum. To characterize the relation between sound absorption and structural strength, we analyzed the thermal-viscous dissipation spectrum, total mechanical energy spectrum, and average stress spectrum, which reveal the mechanism of lowfrequency absorption enhancement as a near-resonance effect. Adequate investigation of the structural vibration modal properties and stress distribution inspired the proposal of an aluminum-rubber composite structure, which has drastic potential to offer more advanced thermal, mechanical, and acoustic performance.

\section{Materials and Methods}

2.1. Structure Model. Our perforated honeycomb-corrugation hybrid structure is shown in Figure 1. It consists of two perforated panels sandwiching a honeycomb-corrugation rectangular core. The geometry of the core influences the energy absorption and the mechanical performance, but the broadband low-frequency sound absorption does not depend on the choice of the core whether it be hexagonal, rectangular, or triangular $[25,26]$. We chose the rectangular core for simplicity. The top panel was periodically perforated to form an MPP, while the bottom is a flexible backing panel. The corrugation core crosses all the honeycomb cavities. The corrugation core was also perforated at the same vertical location where the top panel is perforated.

Figure 2(a) is the top view of the rectangular honeycomb core. The unit cell length is $b_{2}$, and the inner cell length is $b_{1}$ which is also the width of the honeycomb cavities. The wall thickness between the cavity is $t_{w}=b_{2}-b_{1}$. Inside each unit cell, there is a microhole perforated to form an MPP as the top panel. Figure 2(b) is the front view of the Sandwich panel. The diameters of the perforated hole in the top panel and the corrugation panel are $d_{1}$ and $d_{2}$, respectively. The subscripts 1 and 2 represent the top and corrugation panels. The thicknesses of the top and corrugation panels are $t_{1}$ and $t_{2}$, respectively. The thickness of the bottom panel is also $t_{1}$. The corrugation is inclined at an angle $\theta$ and has two holes perforated at the same vertical location where the top panel is perforated.

2.2. Geometry Analysis. Six honeycomb cells were selected to form a unit cell of a periodic array (Figure 3), which is used to characterize the sound absorption performance of the full structure. The red-line crossing cavities in the front view is the corrugation panel. These six cells are equivalent to six Helmholtz resonators paralleling each other and connected together to an MPP.

The geometry parameters used in our model are provided in Table 1.

Figure 4 shows the details of our structure consisting of six honeycomb cells $C_{1}, C_{2}, C_{3}, C_{4}, C_{5}$, and $C_{6}$ with MPP as the top panel, a nonperforation board as the bottom panel, and an inclined corrugation. $C_{1}$ and $C_{4}$ have a height of $H^{\prime}=H-t_{2}$. The horizontal board with a thickness $t_{2}$ (shown in orange) in $C_{4}$ is not perforated. $C_{2}$ and $C_{6}$ are identical, while $C_{3}$ and $C_{5}$ are also identical. The inclined corrugation board inside $C_{2}$ and $C_{3}$ is symmetrical to the part inside $C_{5}$ and $C_{6}$. The vertical location of the perforations inside the corrugation is the same as the top MPP. 


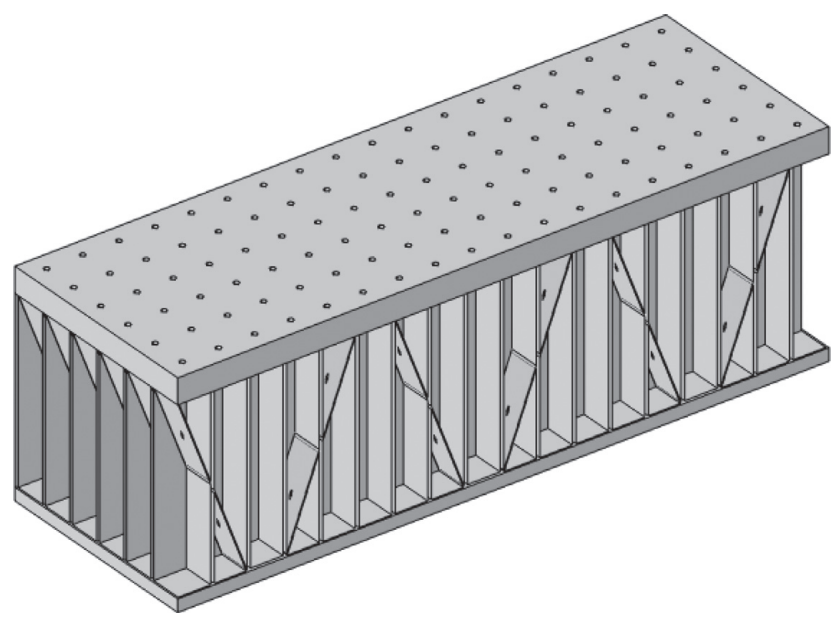

FIGURE 1: Perforated honeycomb-corrugation sandwich panel metamaterial structure.

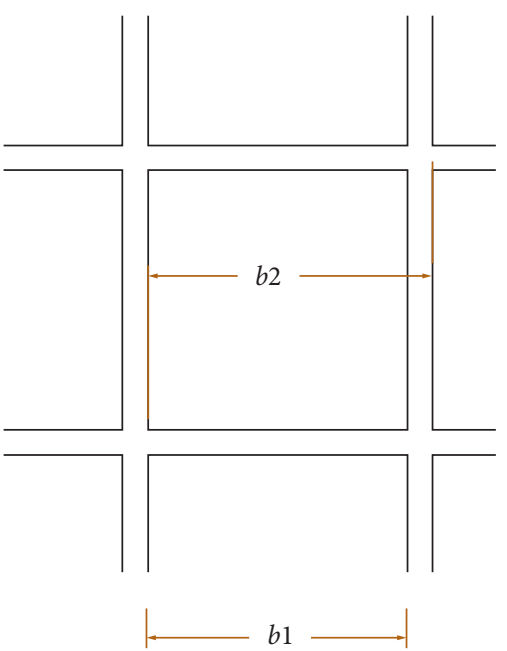

(a)

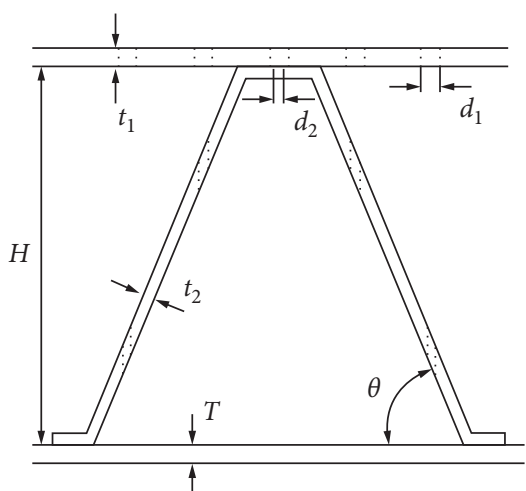

(b)

Figure 2: (a) Top view of the honeycomb core. (b) Front view of the sandwich panel.

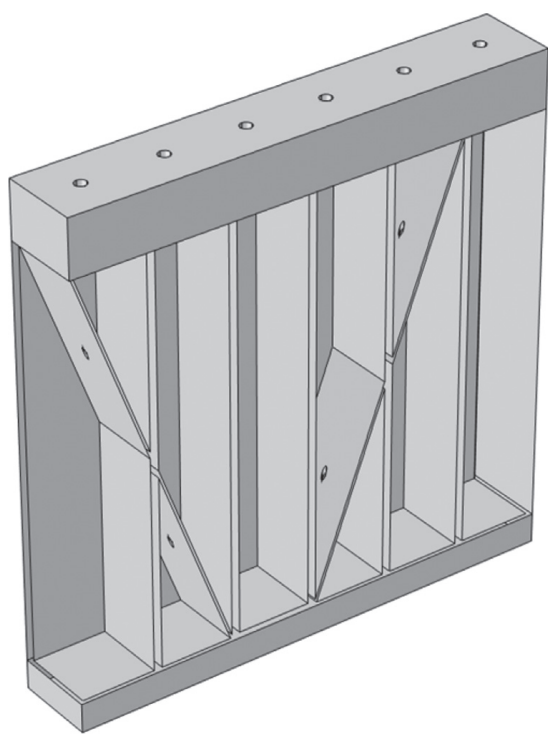

(a)

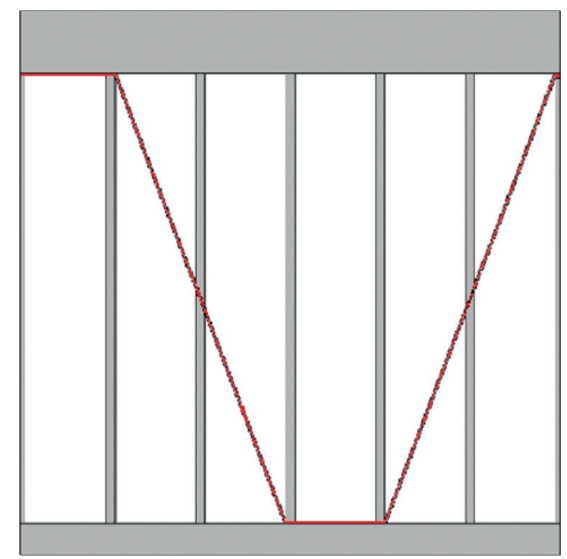

(b)

Figure 3: (a) 3D model of six honeycomb cells. (b) 2D model of six cells. 
TABLE 1: Geometry parameters.

\begin{tabular}{|c|c|c|c|}
\hline Name & Value & Unit & Meanings \\
\hline$b_{1}$ & 3.6 & $\mathrm{~mm}$ & The side length of the air cavity in the honeycomb \\
\hline$b_{2}$ & 3.8 & $\mathrm{~mm}$ & The side length of the honeycomb structure \\
\hline$t_{1}$ & 1.0 & $\mathrm{~mm}$ & The thickness of MPPs in top and bottom panels \\
\hline$t_{2}$ & 1.0 & $\mathrm{~mm}$ & The thickness of the corrugations \\
\hline$t_{\mathrm{w}}$ & 0.4 & $\mathrm{~mm}$ & The thickness of the cavity \\
\hline$t_{\mathrm{c}}$ & 4.0 & $\mathrm{~mm}$ & The thickness of the incident air \\
\hline$d_{1}$ & 1.0 & $\mathrm{~mm}$ & The diameter of the perforation in MPPs \\
\hline$d_{2}$ & 1.0 & $\mathrm{~mm}$ & The diameter of the perforation in the corrugation \\
\hline $\bar{H}$ & 20 & $\mathrm{~mm}$ & Height of the honeycomb \\
\hline$h$ & 1.0 & $\mathrm{~mm}$ & Height of the bottom panels \\
\hline$c_{0}$ & 343 & $\mathrm{~m} / \mathrm{s}$ & Sound speed in air \\
\hline$\rho_{0}$ & 1.213 & $\mathrm{~kg} / \mathrm{m}^{3}$ & The density of the air \\
\hline
\end{tabular}

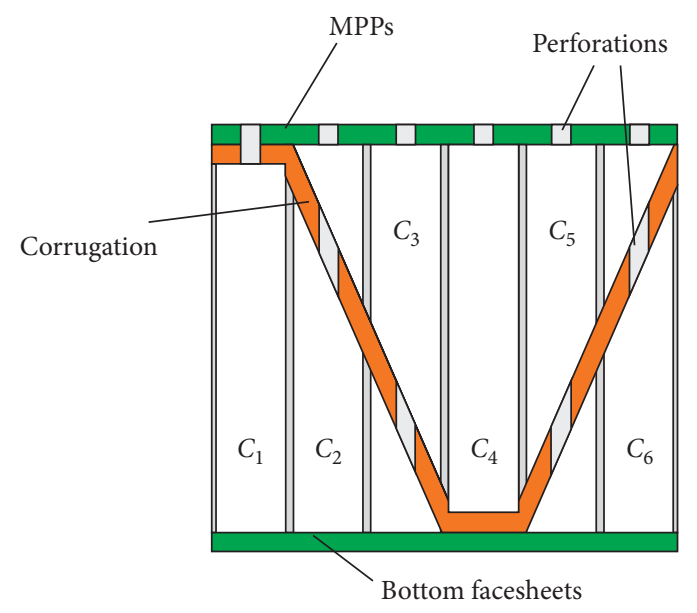

Figure 4: 2D model details of the whole structure.

2.3. Finite Element Model. Finite element modeling and simulation is a commonly used method in structural design, which can promote market competitiveness by saving time and experimental costs $[27,28]$. To investigate the flexible structure's absorption behavior, finite element method (FEM) analysis was performed using COMSOL Multiphysics 5.4. The simulations using COMSOL analyzed the elastic displacement field inside the solid structure and the acoustic pressure distribution in the cavities. From the analyses, we obtained the acoustic impedance and the sound absorption coefficient. The solid structure deformation analysis provides information on the structure strain capacity, which can be used to determine if the flexible structure can be maintained as the honeycomb-corrugation sandwich panel or suffers from irreversible destruction caused by vibration. As shown in Figure 5, the model consists of three parts: the top is a perfectly matched layer that absorbs all the reflection; the middle layer is air in which the incident acoustic wave propagates; and the bottom is the flexible structure.

A plane wave propagating downward in the $z$-direction is excited using a background pressure field. The plane wave amplitude was selected to be the critical sound pressure at
$100 \mathrm{~Pa}$ corresponding to $130 \mathrm{~dB}$, which can generally cause human suffering. For comparison, a jet plane can cause $140 \mathrm{~dB}$ of noise at $100 \mathrm{~m}$. The air in the honeycomb was modeled using a thermoviscous acoustic module. The structure domain was modeled by a solid mechanics linear elastic model with the assumption of small deformations. We chose aluminum and rubber as our candidate structural materials. The aluminum has a density of $2600 \mathrm{~kg} / \mathrm{m} 3$, Young's modulus of $65 \mathrm{GPa}$, and Poisson's ratio of 0.33 at room temperature, while those of rubbers were estimated from commonly used rubber with a density of $1300 \mathrm{~kg} / \mathrm{m} 3$, Poisson's ratio of 0.4 , and Young's modulus of 500, 50, 5, and $0.5 \mathrm{MPa}$, respectively, labeled as No. 1, 2, 3, and 4. Because of the high thermal conductivity of aluminum, its thermalviscous acoustic boundary was set to be isothermal, while it was set to be adiabatic for rubber due to its poor thermal conductivity. Multiphysics coupling was set on the boundary between the acoustic-thermoviscous acoustic boundaries, acoustic-structure boundaries, and thermoviscous acousticstructure boundaries. Considering the mirror symmetry of the whole geometry, only half of the model was calculated, and all the physics was exerted by symmetrical boundary conditions at the cross section. 


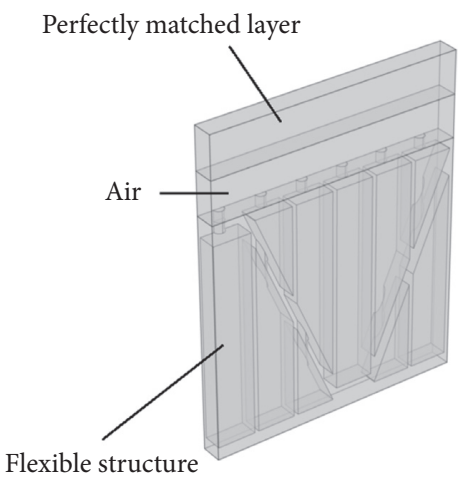

(a)

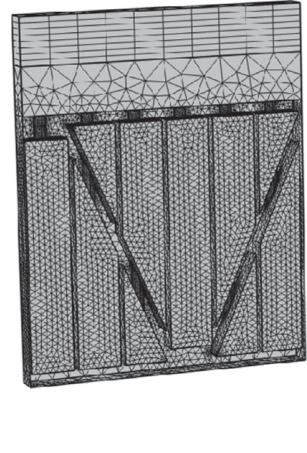

(b)

Figure 5: (a) COMSOL simulation of the flexible structure physical field. (b) Mesh of the model.

The acoustic impedance is given by

$$
z=\frac{\left(p_{t} / v z\right)}{Z_{0}}
$$

where $p_{t}$ is the total acoustic pressure on the incident surface, $v z$ is the normal component of surface sound velocity, and is the surface average. The sound absorption coefficient is calculated as

$$
\alpha=1-\left|\frac{z-1}{z+1}\right|^{2}
$$

The frequency range was $25 \mathrm{~Hz}$ to $2000 \mathrm{~Hz}$ with increments of $25 \mathrm{~Hz}$. The acoustic interface calculated the total acoustic pressure and local sound speed. The solid mechanics interface calculated displacement, mechanical energy, and stress. The thermal interface modeled energy dissipation. All these results were critical in the analysis of the acoustic and mechanical performance of the structure.

\section{Results and Discussion}

3.1. Research on Acoustic-Structure Interaction. In this part, acoustic-structure interaction in our perforated honeycomb hybrid structure made from pure aluminum and rubbers was investigated. To make our study clearer and exhibit a direct comparison with the traditional rigid model, the materials were set no damping so that the dissipation of sound energy was only from the thermal-viscous dissipation of air inside the cavities.

3.2. Sound Absorption of Different Materials. Figure 6 shows the absorption spectrum of each material. The main absorption peaks of aluminum and rubber $1 / 2 / 3 / 4$ were around $1750 \mathrm{~Hz}, 1750 \mathrm{~Hz}, 1400 \mathrm{~Hz}$, and $650 \mathrm{~Hz}$, and the bandwidths of the main absorption peak were about $500 \mathrm{~Hz}$, $450 \mathrm{~Hz}, 450 \mathrm{~Hz}$, and $150 \mathrm{~Hz}$, respectively. The absorption spectrum of rubber 4 has many sharp absorption peaks, among which the lowest frequency absorption peak was around $400 \mathrm{~Hz}$ with a full width at half maximum (FWHM) of about $50 \mathrm{~Hz}$. For a strong sound wave with a pressure of $100 \mathrm{~Pa}$, the absorption spectrum of aluminum and rubber 1 with a modulus of $500 \mathrm{MPa}$ did not differ much, while for rubber materials of $50 \mathrm{MPa}$ and below, as the material modulus decreased, new absorption peaks emerged at a lowfrequency range, and the FWHM decreased significantly. The new peaks benefit low-frequency absorption, while the narrow bandwidth is not in favor of broadband absorption leading to a trade-off consideration. In our case, the optimal modulus is expected to be between $5 \mathrm{MPa}$ and $500 \mathrm{MPa}$, in which low-frequency absorption can be achieved while the absorption bandwidth is not significantly sacrificed.

3.3. Energy Distribution Analysis and Stress Response. To fully understand the influence of different materials on sound absorption, the average thermal-viscous energy dissipation was studied. As is exhibited by Figure 7, the total thermal-viscous dissipation mainly concentrates near the perforation, which satisfies the description of MPP's theory and results from other researchers [23]. Additionally, as is shown in Figure 8, the average thermal-viscous energy dissipation is positively related to frequency, where more energy is dissipated through viscous loss at high frequencies, as expected. The thermal energy dissipation of the aluminum was greater than the viscous energy dissipation at a low frequency under $700 \mathrm{~Hz}$, while the viscous energy dissipation of the rubber was the dominant thermal-viscous energy dissipation. Furthermore, the thermal energy dissipation of aluminum was much greater than that of the rubbers, which can be explained by the discrimination of their thermal conductivity. As for the comparison among the rubbers, a lower modulus led to higher thermal and viscous dissipation at a low frequency $(<1100 \mathrm{~Hz})$. The dissipation spectrum for rubbers 3 and 4 was filled with sharp peaks, similar to the absorption peaks distribution. Compared to a rigid material, the rubber highly suppressed thermal dissipation because of its low thermal conductivity. However, viscous dissipation, which dominates total dissipation, can benefit a lot from the low modulus of rubber to dramatically enhance sound absorption, especially at low frequencies.

The relationship between the modulus and energy dissipation can be understood through mechanical analysis. As shown in Figure 9, with a decrease in the modulus, the positively frequency-related total kinetic energy and total 


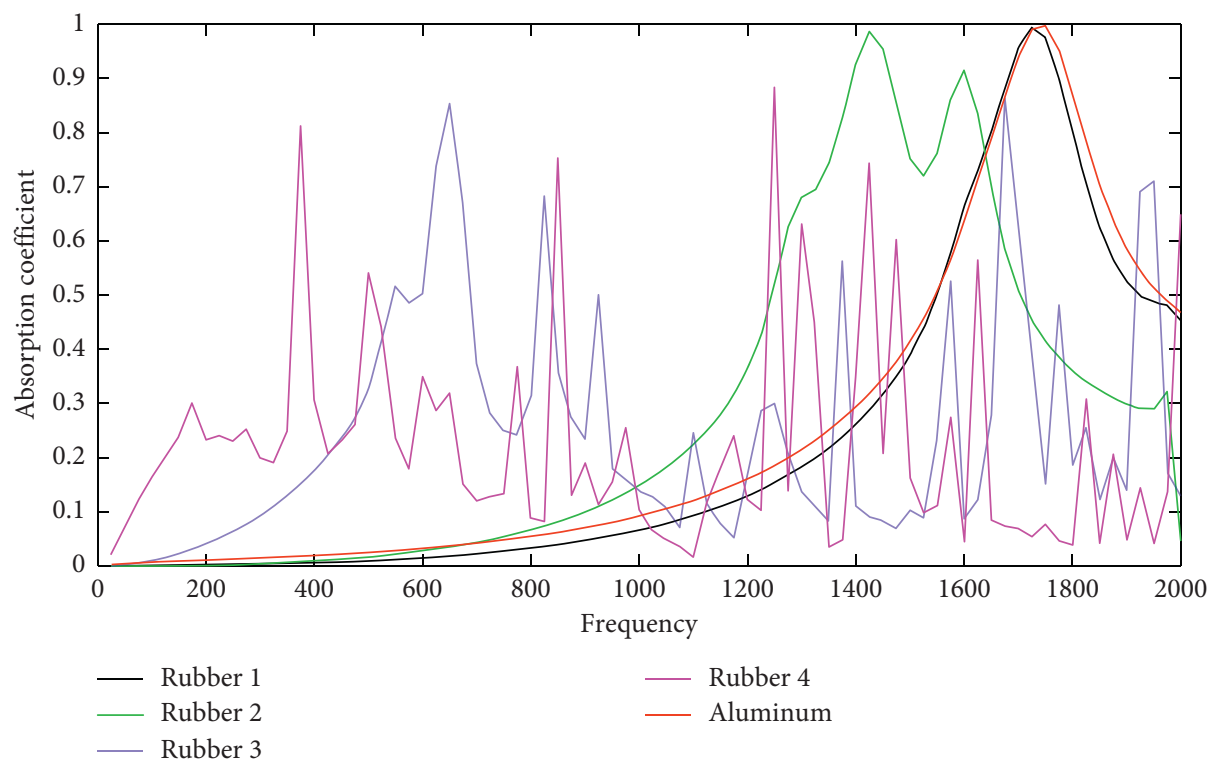

Figure 6: Absorption spectrum of $\mathrm{Al}$ (red), rubber 1 (black), rubber 2 (green), rubber 3 (blue), and rubber 4 (magenta). The unit of the $x$-axis of all the curve figures is $\mathrm{Hz}$.

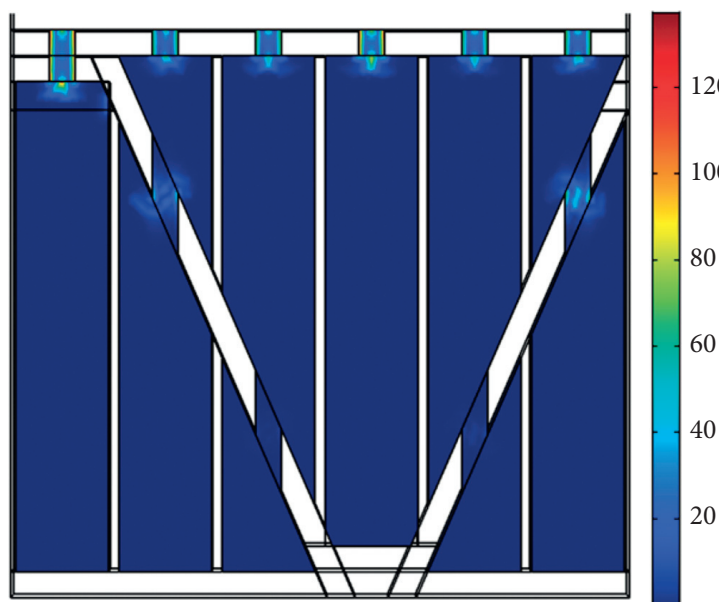

(a)

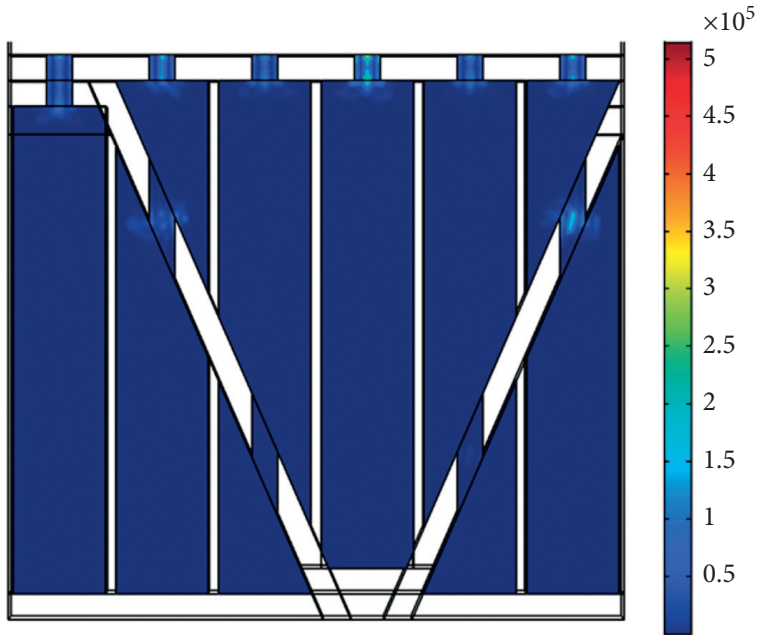

(b)

Figure 7: Total thermal-viscous dissipation of rubber 2 at (left) 105 and (right) $1475 \mathrm{~Hz}$.

elastic strain energy got closer. For the rubbers, they were equal beyond a certain threshold frequency. This phenomenon can be intuitively explained by a $1 \mathrm{D}$ forced harmonic oscillator with a steady-state solution $A \cos (\omega t)$ where the kinetic energy is $1 / 2 m \omega A^{2}$ and the elastic energy is $1 / 2 m \omega_{0} A^{2}$, which are equal at resonance with $\omega=\omega_{0}$. For our system, the damping stems from thermal-viscous dissipation, and the external force is from the incident acoustic wave. The effective stiffness is generally lower if Young's modulus decreases, resulting in a smaller resonant frequency. If the frequency of the external acoustic field is below but near the resonant frequency of the structure, the phase lag of structure vibration increases with the external frequency, and the relative displacement between the air and structure is enhanced, producing a stronger dissipation. After the resonance is achieved, regardless of the air or the structure, the response curve manifests a series of sharp peaks, which are unfavorable in forming broadband. Another negative effect of resonance is severe structural stress, as shown in Figure 10. Compared to the near-resonance region, the average stress in the solid structure would reach one order of magnitude higher in the resonating region, which would be dangerous as this can cause structural failure and is a potential threat to structural health. Consequently, the best choice is to make the structure work right below but sufficiently near the threshold resonant frequency to 


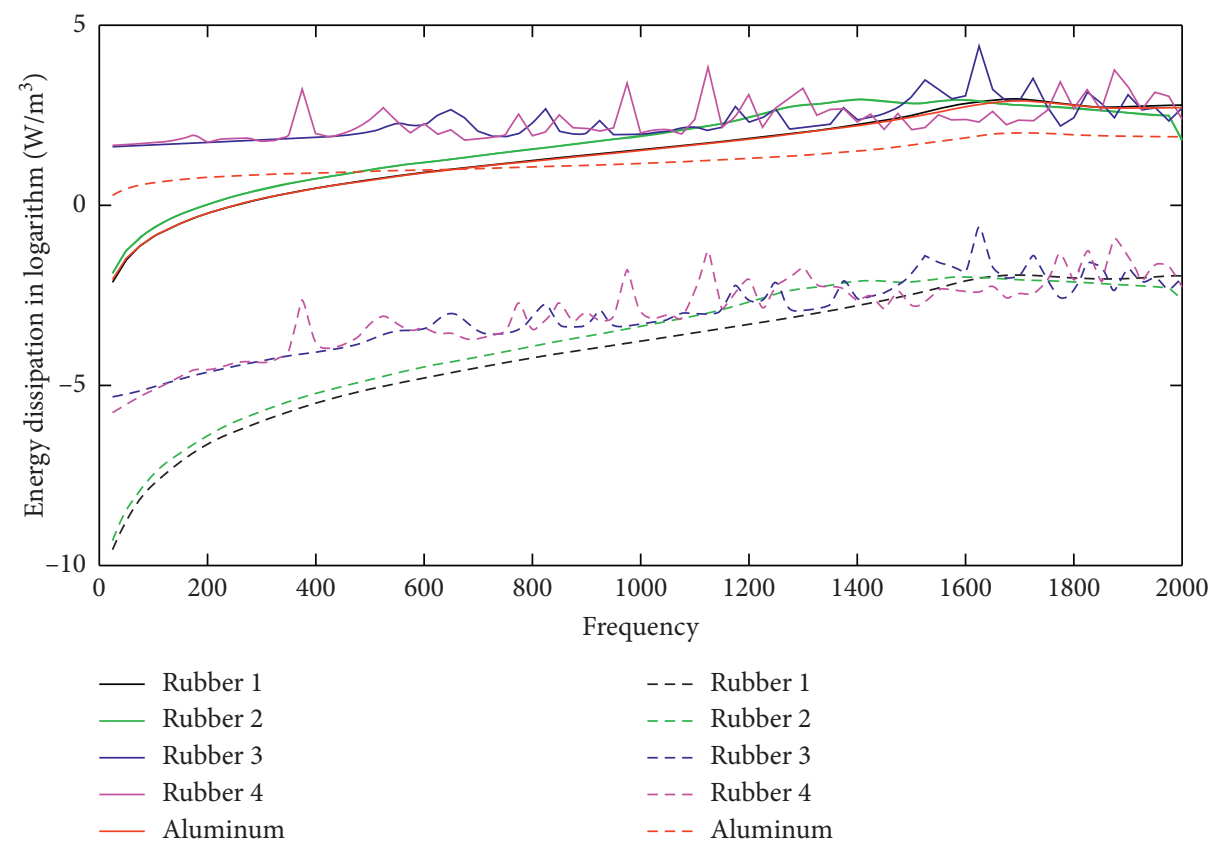

Figure 8: The thermal (dashed line) and viscous energy dissipation of five materials.

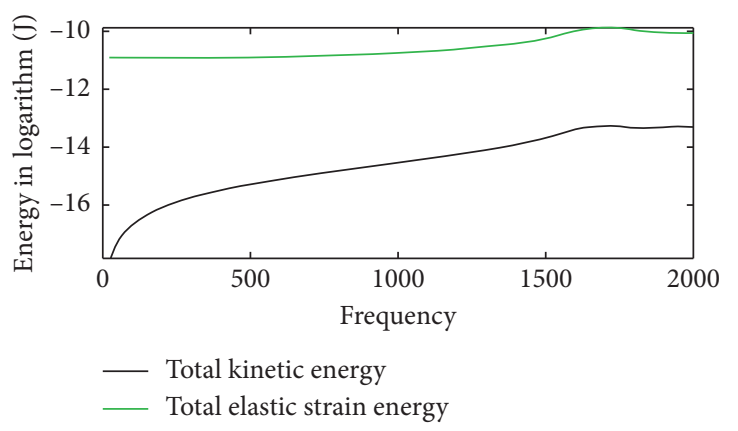

(a)

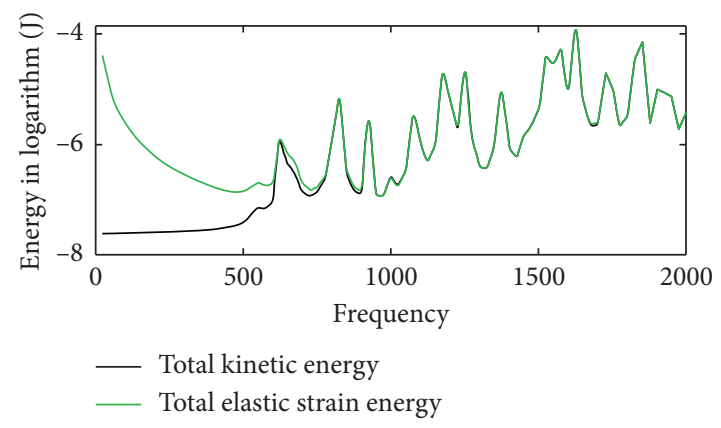

(c)

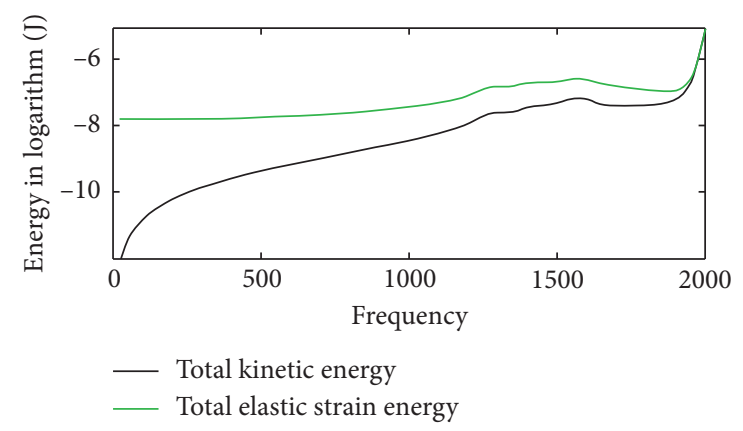

(b)

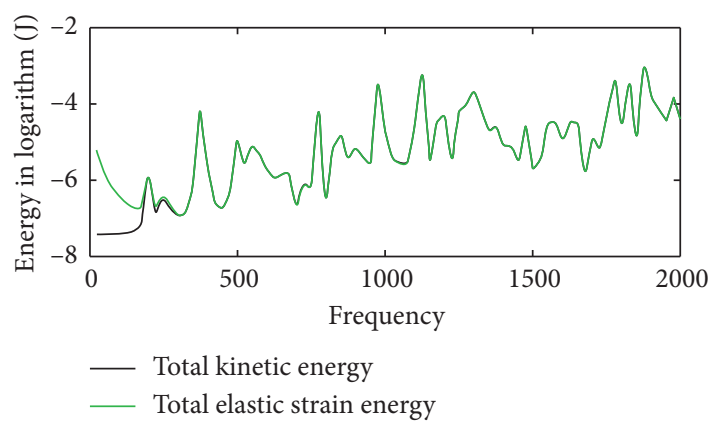

(d)

FIGURE 9: Mechanical energy spectrum of four materials.

simultaneously achieve broadband lower-frequency absorption and to protect the structure from the threat of resonance.

3.4. Structural Vibration Modal Properties. The deformation and stress of different frequencies are shown in Figure 11, which were used to investigate the interaction between the acoustic field and structure. Firstly, due to the corrugation framework segment, these boards can be considered as membranes with different lengths and contribute to a variety of vibration modes and eigenfrequencies. For instance, the vibration of rubber 2 at $500 \mathrm{~Hz}$ was at a low order and mainly 


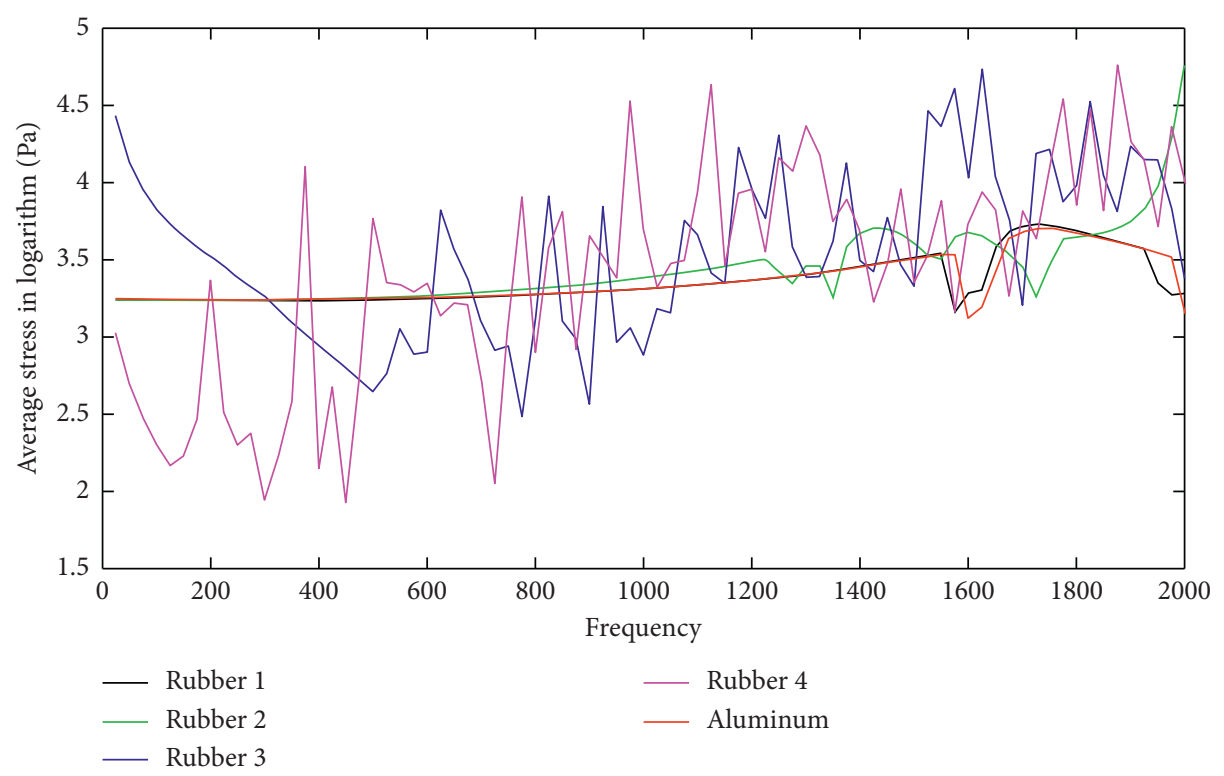

FIgURE 10: Absolute value of average von Mises stress.

concentrated on the longer part of the cavity board; however, the vibration became a high order at $1500 \mathrm{~Hz}$ and $2000 \mathrm{~Hz}$ and transferred to the shorter part. This explains the variety of resonant peaks. Secondly, comparing the vibration modes at the same frequency among different materials showed that the higher-order vibration mode is more likely to be excited in structures with a lower modulus, which tends to deform the structure locally with higher stress. Recalling the results from Figure 10 that the average stress will increase if the material modulus is lowered, it can be concluded that a lower modulus is unfavorable for releasing and uniformly distributing the vibration stress, thus causing a potential threat to structural health. Last but not least, compared to the vertical-cavity board of the honeycomb, corrugation and perforated panels exhibit insignificant vibration under the acoustic field.

3.5. Design of the Composite Structure. According to the discussion above, we have revealed that the low-frequency sound absorption performance of our structure can be improved by using appropriate low-modulus material to take the advantage of acoustic-structure interaction which is mainly contributed by the vibration of cavity board. However, the structure with low modulus produced unsatisfying mechanical performance. Therefore, we proposed a novel composite structure to combine the advantages of high- and low-moduli materials altogether. This composite structure consists of an aluminum framework, that is, the corrugation and MPPs, and the rubber-boarded honeycomb cavities. Recalling that the sound absorption performance of rubber 1 was almost equivalent to that of aluminum, we adopted rubbers 2,3 , and 4 as the lowmodulus component. As a practical structure design, in this part, the damping of the structure material was considered through the isotropic structural loss factor $\eta$ which changed
Young's modulus $E$ from a real number to a complex one $(1+i \eta) E$.

3.5.1. Sound Absorption. The sound absorption spectrum of pure rubber 2 and aluminum-rubber 2 composite structure with different $\eta$ is shown in Figure 12. Firstly, it can be observed that the composite structure performed almost the same sound absorption ability as the corresponding pure rubber structure, which verifies our argument that the vibration of the framework contributes little to sound absorption. Secondly, comparing the same aluminum-rubber 2 composite structure with different $\eta$, the low-damping case with $\eta=0.4$ differed little in the frequency and strength of main absorption peak with a no-damping case but greatly broadened the absorption band from $450 \mathrm{~Hz}$ to more than $900 \mathrm{~Hz}$. As for the higher-damping case with $\eta=1.4$, a relatively significant $200 \mathrm{~Hz}$ shift of the main absorption peak can be observed. Then, for different aluminum-rubber composite structures with the same $\eta=0.4$, rubber 3 gave the best low-frequency and broadband absorption performance with the main absorption peak at $700 \mathrm{~Hz}$ and a bandwidth of $1750 \mathrm{~Hz}$ (recall that the original rigid aluminum structure has the absorption peak at $1700 \mathrm{~Hz}$ and a bandwidth of only $400 \mathrm{~Hz}$ ). As for rubber 4, though the absorption peak could reach even $150 \mathrm{~Hz}$, the absorption strength and bandwidth dropped significantly, which was similar to the no-damping situation discussed in the first part. In summary, with a balanced selection of material (in our case, rubber 3 ), our composite structure possesses an advanced low-frequency sound absorption performance.

Furthermore, the energy conservation and partition during the absorbing process are studied. In general, the acoustic wave transports from exterior space to the top perforation panel and causes the vibration of the structure (to be more specific, in our composite structure, the 

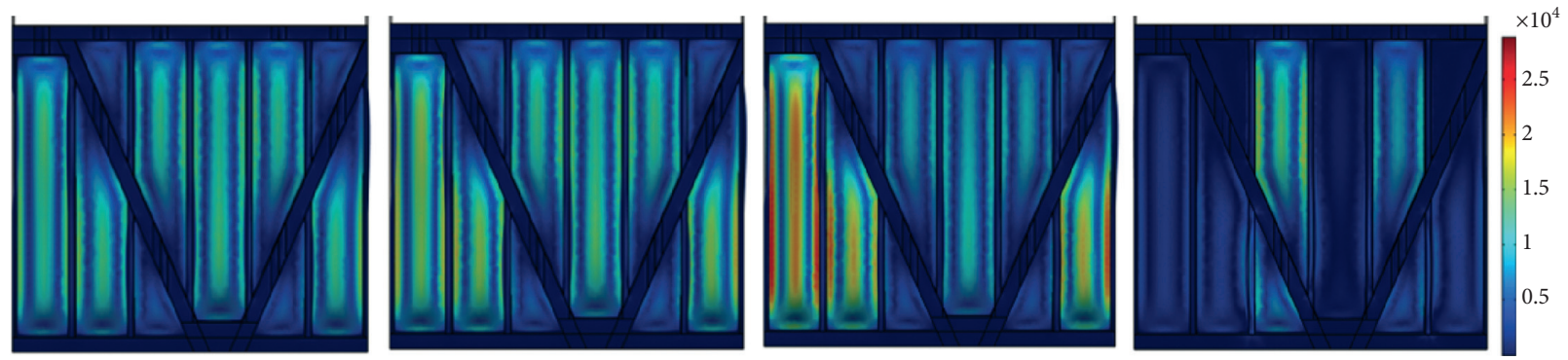

(a)
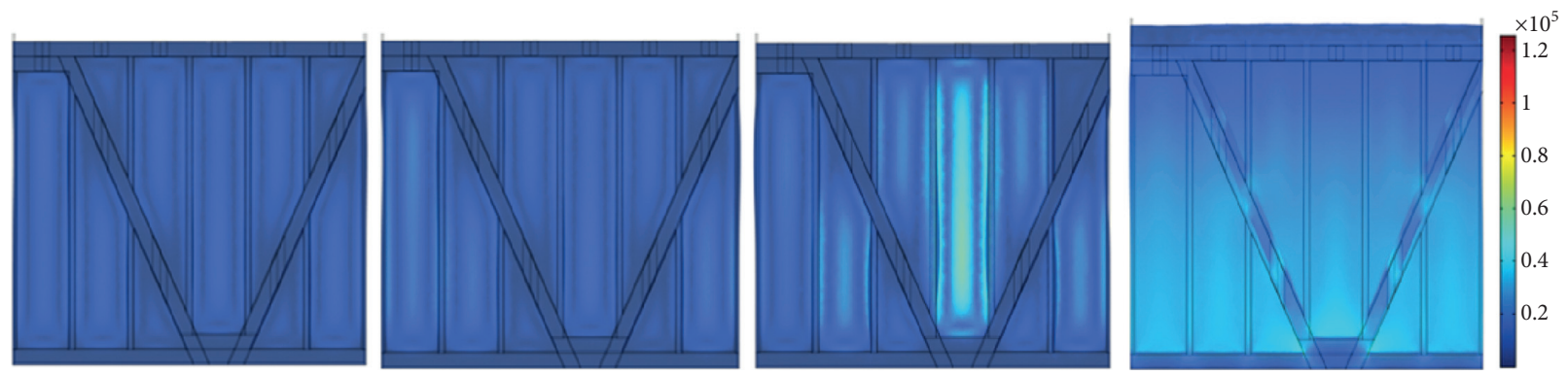

(b)
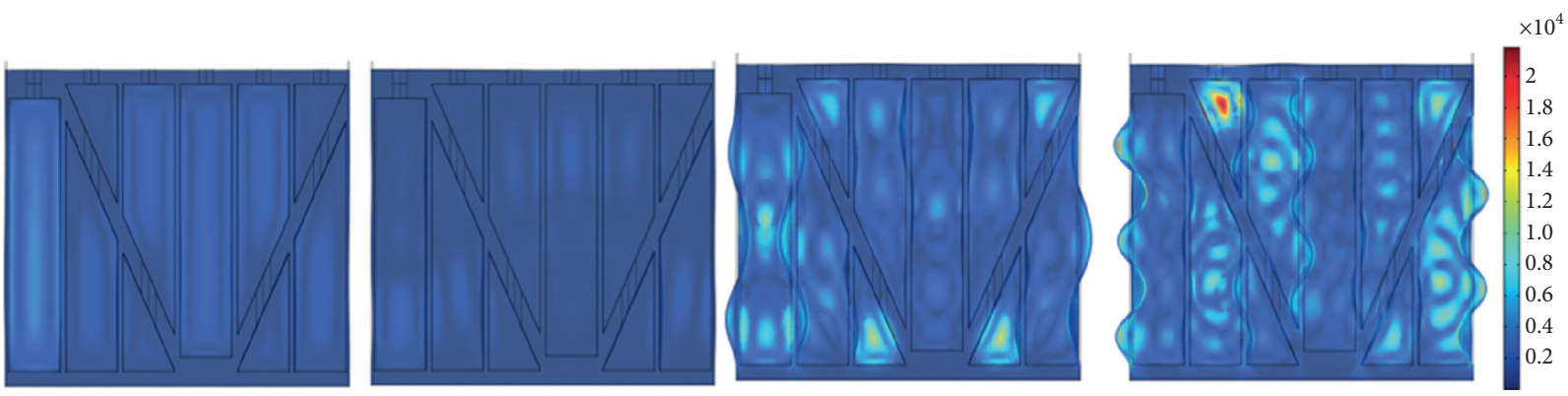

(c)
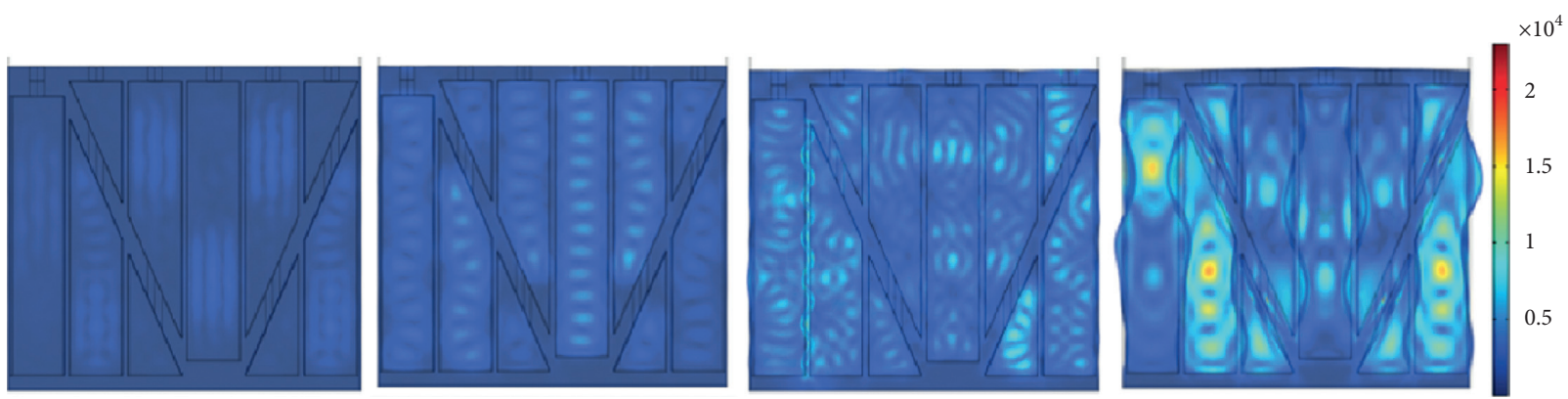

(d)

Figure 11: Structure deformation (amplified) and stress modes of each material at 500, 1000, 1500, and $2000 \mathrm{~Hz}$.

vibration of cavity board) and the air in the cavity. The mechanical vibration transforms and stores the acoustic energy as kinetic energy and elastic strain energy. Besides, the vibration needs to do work to overcome the damping force determined by factor $\eta$, resulting in the mechanical dissipation. The acoustic vibration in the cavity is also resisted by viscous force and thus contributes thermal-viscous dissipation. The power spectra of these four energies of aluminum-rubber 2 composite structure are plotted as stacked bars while the total acoustic energy flowed into the structure, which is calculated by the integral of the normal acoustic intensity at the incident surface, is plotted as black dashed lines in Figure 13. The energy conservation can be verified by the equivalent between the total input acoustic energy and the summation of four other kinds of energies. Besides, the curve of the total energy spectrum has almost the same shape as the curve of the absorption coefficient in Figure 12. As for the proportion of these four energies, the mechanical dissipation dominates in the whole band. The second most important contribution is from thermal- 


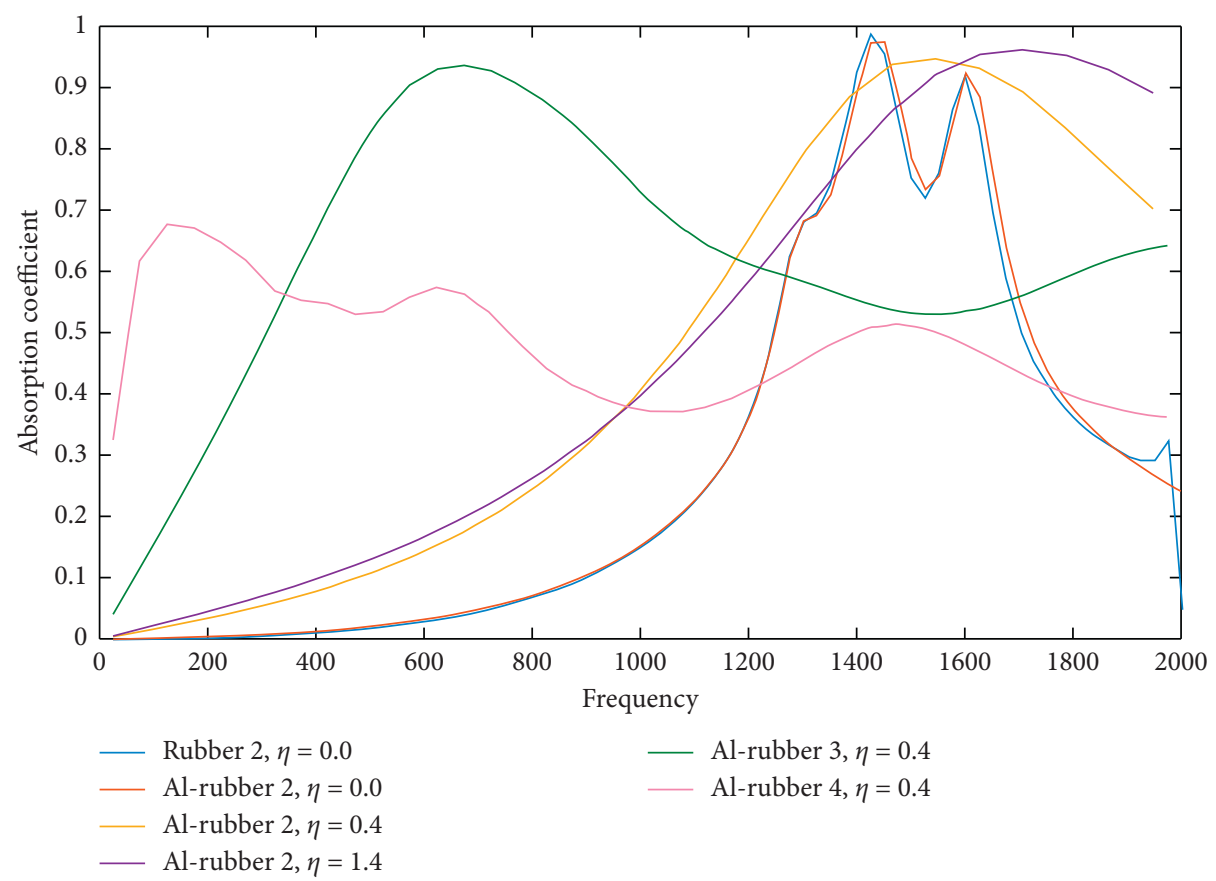

FIgURE 12: Absorption spectrum of different structures.

viscous dissipation but it has a near-plat region from $1400 \mathrm{~Hz}$ to $1950 \mathrm{~Hz}$, which is just the main absorption peak and the whole structure reached a near-resonate state. On the contrary, the kinetic energy becomes significant only in that region but contributed little in else. According to the discussion of Figure 8 in the first part, the thermal-viscous dissipation mostly concentrates near the perforation which is made of aluminum and vibrates little. Therefore, once the air in the cavity reached its resonance, the thermal-viscous dissipation could no longer be further enhanced, resulting in the saturation and the flat curve in 1400-1950 Hz. According to the above discussion of Figure 9, the increase of kinetic energy indicated the approach to near-resonance. Therefore, as a conclusion, it is the mechanical vibration of the flexible cavity board that gave the most important contribution in our composite structure to sound absorption.

The study on stress and deformation mode is shown in Figure 14. Basically, the pure and composite structure gave the same vibration mode. Their stress and deformation were at the same level as a similar distribution except for the stress concentration on the aluminum framework in composition structure, which was caused by a sharp change of mechanical properties at the joint of aluminum and rubber. This stress concentration may bring a challenge to manufacture because the honeycomb and corrugation are traditionally glued together; thus, the joint part is somehow weak.

3.5.2. Mechanical Performance. Besides advanced sound absorption ability, the composite structure was demonstrated to be able to retain the most of the great mechanical performance of the rigid aluminum structure and is much better than the pure rubber one. Firstly, according to
Figure 15, after exerting the same shear force on the top panel along the width direction, the composite structure deformed a little bit severer than pure aluminum structure but one order of magnitude smaller than the pure rubber one. The stress of the composite structure mainly concentrated near the perforation part. Secondly, as exhibited in Figure 16, under a normal compression pressure on the top panel, the composite structure exhibited the same order of magnitude of deformation as the aluminum structure. The stress concentration regions were mainly distributed on the top aluminum panel, avoiding the relatively weak conjunction part between the honeycomb cavity board and corrugation board. In another test, a normal compression displacement was exerted on the top of the panel, and the results are shown in Figure 17. The stress on the rubber part was relatively lower in either the pure rubber or composite structures than that exerted on the aluminum. However, due to the support from the rigid framework, the composite structure was capable of withstanding similar stress to that exerted on the aluminum structure. Hence, compared to the rubber structure, the composite structure exhibited a higher stiffness and a stronger ability to resist normal compression, which is essential for the structure to stay healthy and avoid being damaged by external forces.

Except for the great mechanical performance, the composition structure was also thermally stable. After being heated from $300 \mathrm{~K}$ to $500 \mathrm{~K}$, the thermal expansion and stress, as shown in Figure 18, suggested that the composite structure deformed only slightly, which was similar to the performance of the aluminum one, unlike the significant expansion in the pure rubber structure. Additionally, due to the lower mass density of rubber than that of aluminum (typically a half lower), the composite structure can be lighter than the original pure aluminum structure. In the 


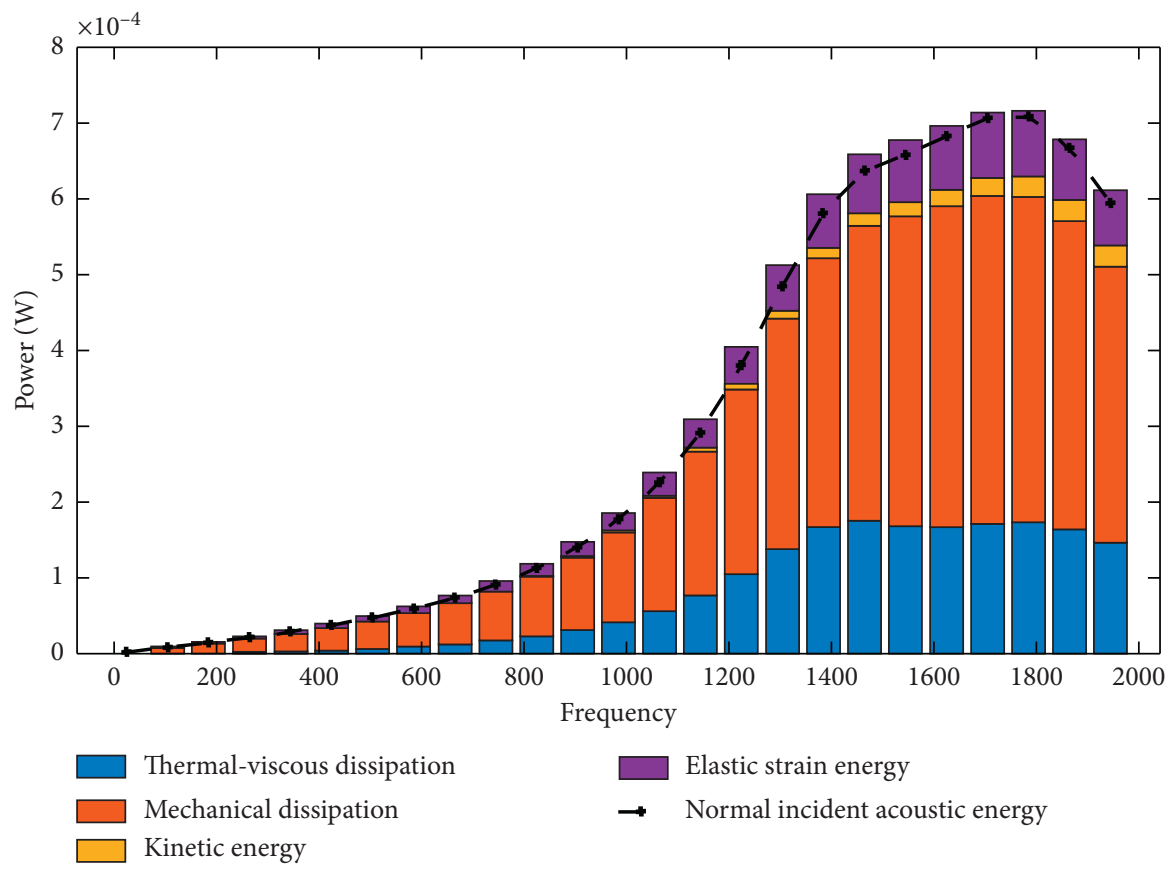

FIGURE 13: Energy spectrum of half of Al-rubber 2 composite structure.
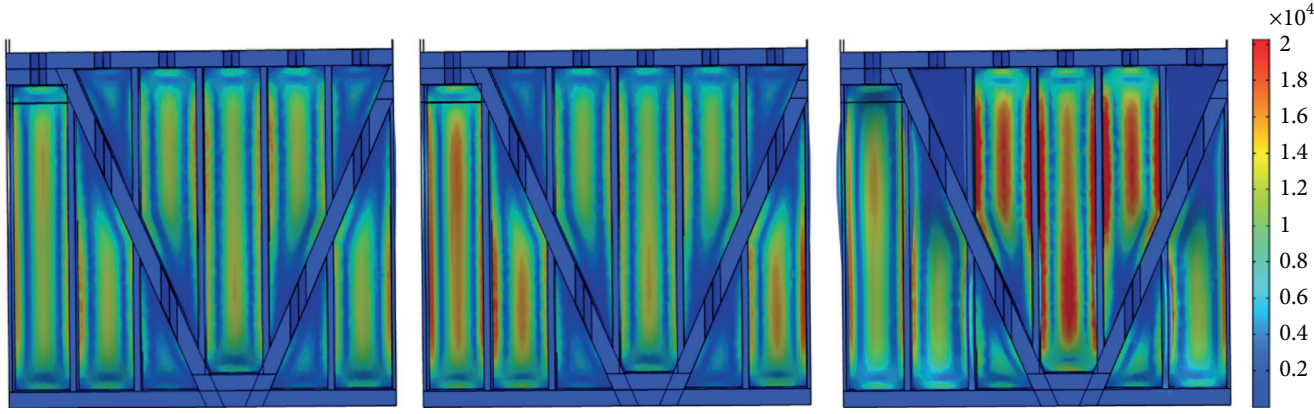

Rubber 2
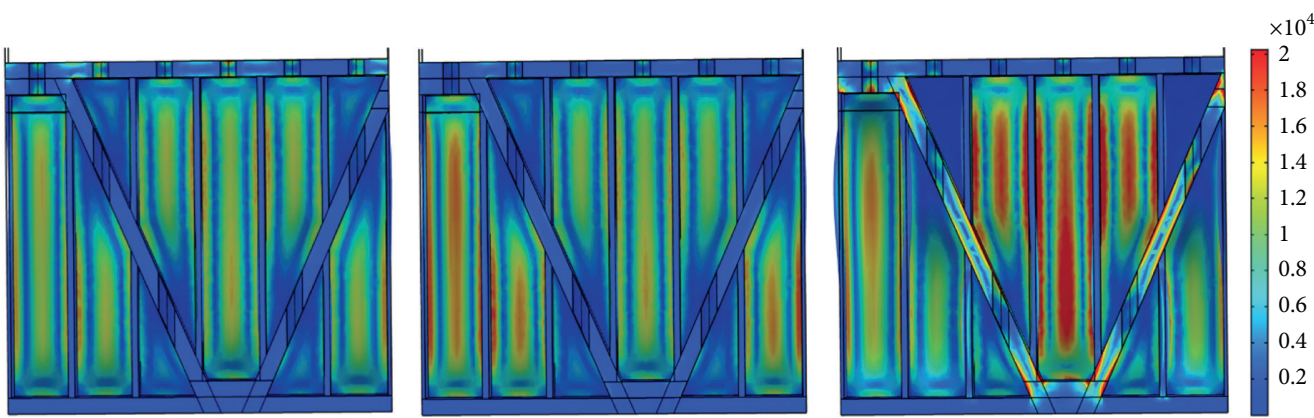

Aluminum-rubber 2

Figure 14: Structure deformation (amplified) and stress modes of each pure (upper) and composite rubber 2 structure at 25, 985, and $1465 \mathrm{~Hz}$ (left to right).

case of rubber 2, one unit of the aluminum structure is $0.9102 \mathrm{~g}$ while that of the composite structure is $0.6973 \mathrm{~g}$, which is $23.3 \%$ lighter. Furthermore, rubber is generally cheaper than aluminum, resulting in the reduction of construction cost.
In summary, on the one hand, compared to a rigid structure made purely from aluminum, the composite structure has a lighter weight and still retains a high resistance to an external load and thermal expansion, which is much better than that of the pure rubber case. On the other 


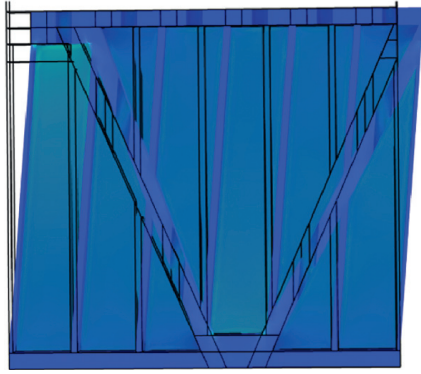

(a)

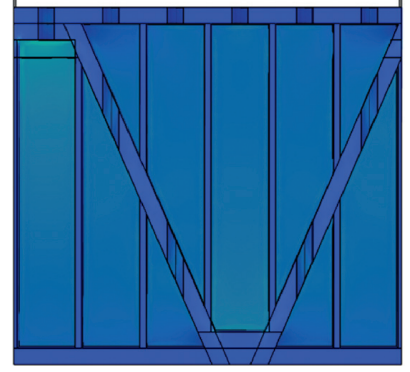

(b)

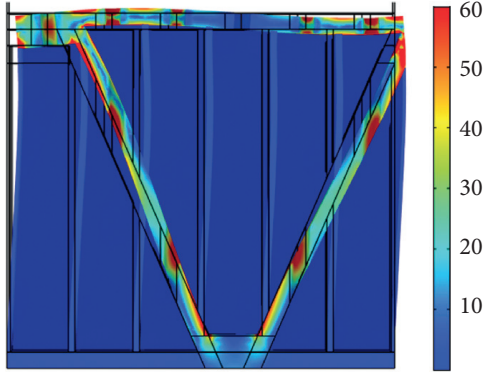

(c)

Figure 15: The steady-state stress distribution after being subjected to the same shear load. The deformation is enlarged $1 \times 10^{7}, 1 \times 10^{8}$, and $1 \times 10^{8}$ times from (a) to (c), respectively.

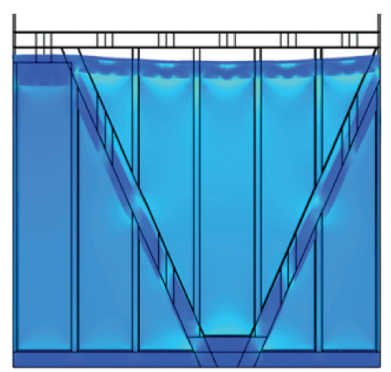

(a)

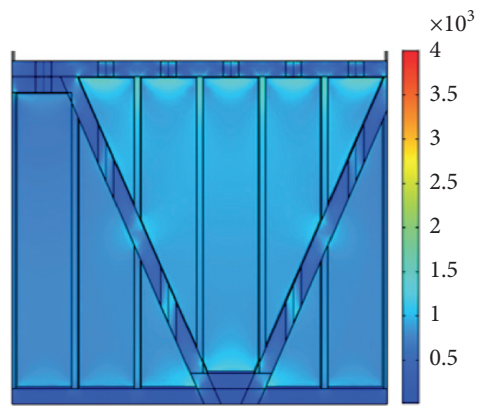

(b)

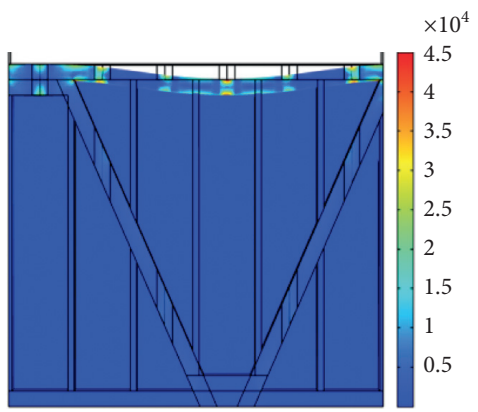

(c)

Figure 16: The steady-state stress distribution after being subjected to the same normal pressure. The deformation is enlarged 5000, 50000, and 50000 times from (a) to (c), respectively.

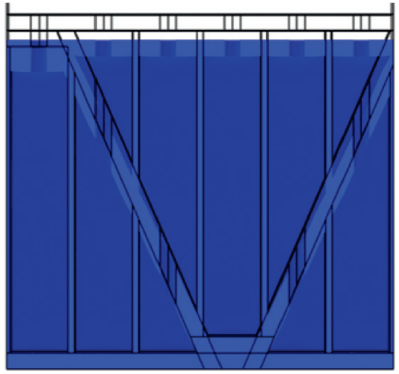

(a)

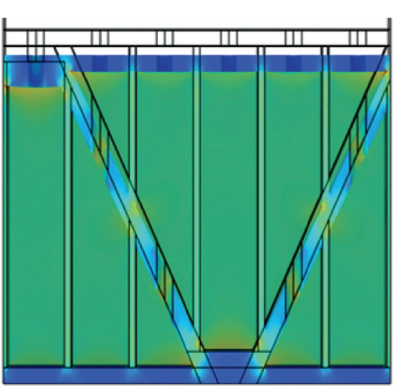

(b)

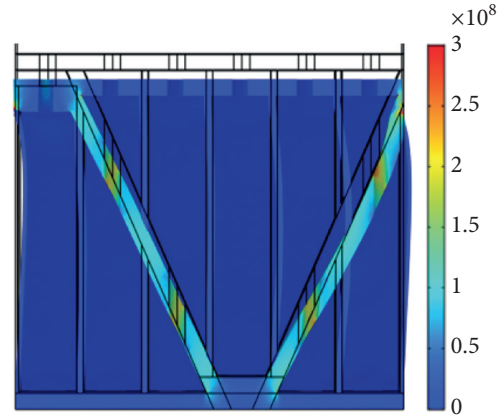

(c)

FIgURE 17: The steady-state stress distribution after being subjected to the same normal compressive strain. The deformation magnifies 3000 times.

hand, the enhancement in the low-frequency sound absorption ability is at the same level as a pure rubber case. It can be concluded that our novel composition structure combining rigid and flexible materials fuses the advantages of the two materials.

The novelty and advantage of our design can be further addressed by comparison with recently reported research. As for the multicavity metamaterials, Tang et al. [23] studied the purely rigid perforated honeycomb-corrugation hybrid sandwich panel. With similar geometry parameters, our composite structure possesses a much lower absorbing peak and broader absorbing width (around $600 \mathrm{~Hz}$ and $1300 \mathrm{~Hz}$ for our rubber 2 case while $1500 \mathrm{~Hz}$ and $600 \mathrm{~Hz}$ for Tang's, resp.). Another structure with symmetrical-coiled-up spaces gave quite low absorbing frequency around $200-300 \mathrm{~Hz}$ but a narrow bandwidth around $100 \mathrm{~Hz}$ [29]. Wu et al. [30] also proposed a perforated panel structure with two absorption peaks at $352 \mathrm{~Hz}$ and $600 \mathrm{~Hz}$ and a $550 \mathrm{~Hz}$ bandwidth but it sacrificed the structure thickness which reached $65 \mathrm{~mm}$, more than 3 times our structure. A similar situation occurred in the multilayer resonator with extended neck proposed by Guo et al. [31] which realized a $550-700 \mathrm{~Hz}$ near-perfect sound absorption but with a thickness of $61 \mathrm{~mm}$. As for the rigid-elastic composite structure, 


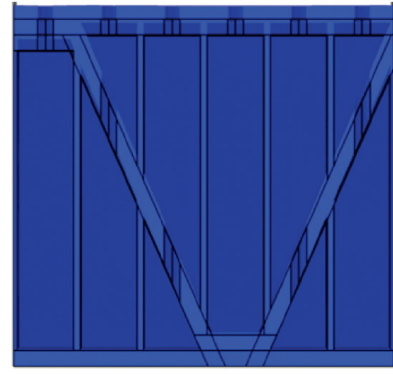

(a)

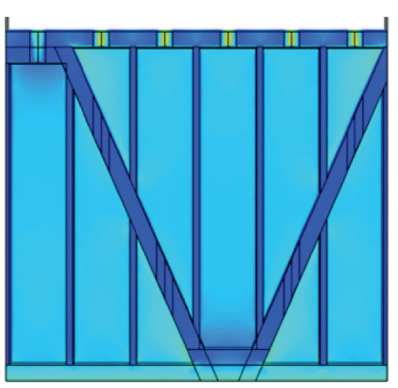

(b)

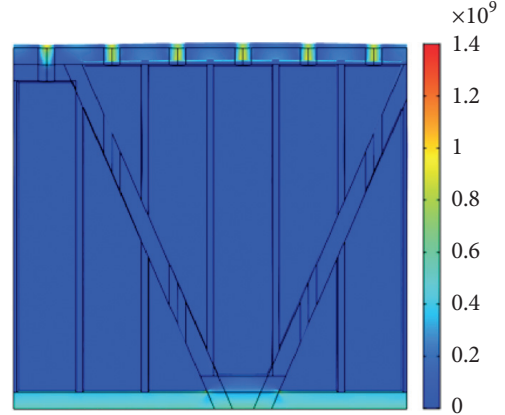

(c)

Figure 18: The steady-state stress and deformation distribution after being heated from $300 \mathrm{~K}$ to $500 \mathrm{~K}$.

Bucciarelli et al. [32] reported an application of elastic membrane on Helmholtz resonator-type absorbing material with a very low-frequency absorbing peak at around $300 \mathrm{~Hz}$ while with a narrow absorbing bandwidth around $200 \mathrm{~Hz}$. Hou et al. [33] researched the rigid MPP with an elastic backboard. Their results do confirm the enhancement from the application of elastic component to low-frequency absorption in the form of generating new low-frequency peak in rigid MPP but also narrow the absorption band, which agrees well with our findings. However, only introducing elastic backboard into rigid MPP exhibits limited enhancement. Even with a much beneficial geometry $(0.3 \mathrm{~mm}$ perforation diameter which is less than half of ours and $40 \mathrm{~mm}$ cavity thickness which is twice ours), the elastic backboard MPP only has a similar performance to our design.

In conclusion, to make better control of noise and meanwhile possess an excellent mechanical property, the acoustic metamaterial should combine the rigid and elastic components together. The former can benefit the low-frequency sound absorption with mechanical dissipation and enhanced thermal-viscous dissipation while the latter provides mechanical stability to the external force. Besides, the rigid part can also be well designed to segment the elastic part into different sizes, which offers more different resonate frequencies and is beneficial to wide-band absorption. Based on these principles, our aluminum-rubber (i.e., rigid-elastic) composite perforated honeycomb-corrugation hybrid sandwich panel exhibits both advanced low-frequency, wide-band sound absorption ability, and high mechanical performance.

\section{Conclusions}

In this paper, we conducted a comprehensive FEM investigation on the acoustic and mechanical performance of a flexible perforated honeycomb-corrugation hybrid Sandwich panel structure made from aluminum and rubbers with different Young's moduli under a 130-dBstrong sound wave. The sound absorption spectrum indicates that reducing moduli creates more low-frequency absorption peaks while narrowing each peaks' bandwidth. In our case, the traditional aluminum structure had a main absorption peak of around $1750 \mathrm{~Hz}$, and the rubber with a modulus of $500 \mathrm{MPa}, 50 \mathrm{MPa}$, and $5 \mathrm{MPa}$ had a main absorption peak at about $1750 \mathrm{~Hz}, 1400 \mathrm{~Hz}$, and $650 \mathrm{~Hz}$, respectively. The bandwidth of aluminum and rubbers was $500 \mathrm{~Hz}, 450 \mathrm{~Hz}, 450 \mathrm{~Hz}$, and $150 \mathrm{~Hz}$, respectively. The spectra for low-moduli materials like the $5 \mathrm{MPa}$ rubber were filled with sharp peaks. Further analysis suggested that thermal-viscous dissipation can be enhanced by a smaller modulus. From the additional analysis on total kinetic and elastic strain energy spectra, average stress spectra, and structural vibration modal, we can infer that the optimal modulus is supposed to be able to keep the structure working at a near-resonate state to obtain higher absorption performance and guaranteed structural health. Based on our findings, a novel aluminum-rubber composite structure was proposed, and our simulations proved its combination of the advantages of both aluminum and rubber. The composite structure is lighter and could achieve an enhanced low-frequency sound absorption ability (in our case, compared with rigid aluminum structure, a $1000 \mathrm{~Hz}$ lower absorption peak and 4 times wider absorbing bandwidth) and possess a strong resistance to thermal expansion, normal and shear load. Our research contributes to the better control of noise and vibration through metamaterials.

\section{Data Availability}

The data that support the findings of this study are available from the corresponding author upon reasonable request.

\section{Conflicts of Interest}

The authors declare that they have no conflicts of interest regarding the publication of this paper.

\section{Acknowledgments}

This work was supported in part by the National Natural Science Foundation of China under Grant 61774049, the National Key R\&D Program of China under Grant no. 2019YFE0120700, and the Shanghai Municipal Commission of Economy and Informatization under Grant PKX2020D10. 


\section{References}

[1] Z. Fang, B. Zhang, and W. Li, Noise Control Engineering, China Science Publishing \& Media, Beijing, China, 2013.

[2] D. He, Z. Guo, H. Liao et al., "Research progress and development trend of porous sound-absorbing materials," Materials Reports, vol. 26, no. S1, pp. 303-306+333, 2012.

[3] Y. Yang, B. Li, Z. Chen et al., "Sound insulation of multi-layer glass-fiber felts: role of morphology," Textile Research Journal, vol. 87, no. 3, pp. 261-269, 2017.

[4] D. Oliva and V. Hongisto, "Sound absorption of porous materials-Accuracy of prediction methods," Applied Acoustics, vol. 74, no. 12, pp. 1473-1479, 2013.

[5] M. Yang and P. Sheng, "Sound absorption structures: from porous media to acoustic metamaterials," Annual Review of Materials Research, vol. 47, no. 1, pp. 83-114, 2017.

[6] D.-Y. Maa, "Potential of microperforated panel absorber," The Journal of the Acoustical Society of America, vol. 104, no. 5, pp. 2861-2866, 1998.

[7] D.-Y. Maa, "Theory and design of microperforated-panel sound-absorbing construction," Scientia Sinica, vol. 18, pp. 55-71, 1975.

[8] G. Li and C. K. Mechefske, "A comprehensive experimental study of micro-perforated panel acoustic absorbers in MRI scanners," Magnetic Resonance Materials in Physics, Biology and Medicine, vol. 23, no. 3, pp. 177-185, 2010.

[9] K. Sakagami, M. Morimoto, and M. Yairi, "Application of microperforated panel absorbers to room interior surfaces," International Journal of Acoustics and Vibrations, vol. 13, pp. 120-124, 2008.

[10] T. Cox and P. D'Antonio, "Acoustic absorbers and diffusers," in Theory, Design, and Application, CRC Press, Boca Raton, FL, USA, 3rd ed. edition, 2016.

[11] Z. Liu, C. T. Chan, and P. Sheng, "Analytic model of phononic crystals with local resonances," Physical Review B, vol. 71, Article ID 014103, 2005.

[12] N. Fang, D. Xi, J. Xu et al., "Ultrasonic metamaterials with negative modulus," Nature Materials, vol. 5, no. 6, pp. 452-456, 2006.

[13] A. Sukhovich, L. Jing, and J. H. Page, "Negative refraction and focusing of ultrasound in two-dimensional phononic crystals," Physical Review B, vol. 77, Article ID 014301, 2008.

[14] Q. Liu, L. Han, and S. Guan, "Simulation study on the effect of adhesive on the local deformation of honeycomb panel," Materials Science and Technology, vol. 7, no. 3, pp. 89-92+96, 1999.

[15] Z. Fan, Y. Sun, Y. Duan, Z. Guo, and S. Rao, "The influence of metal honeycomb panel parameters on its heat transfer performance," Materials Reports, vol. 27, no. 8, pp. 147-151, 2013.

[16] Z. Wang, "Lightweight honeycomb sandwich structure composite sound," Insulation Material, Noise and Vibration Control, no. 3, pp. 23-25, 1993.

[17] L. J. Gibson and M. F. Ashby, Cellular Solids: Structure and Properties, Cambridge University Press, Cambridge, 1999.

[18] J. Pan, J. Guo, and C. Ayres, "Improvement of sound absorption of honeycomb panels," in Proceedings of Annual Conference of the Australian Acoustical Society, pp. 9-11, Busselton, Western Australia, November 2005.

[19] Y. Tang, F. Li, F. Xin, and T. J. Lu, "Heterogeneously perforated honeycomb-corrugation hybrid sandwich panel as sound absorber," Materials and Design, vol. 134, pp. 502-512, 2017.
[20] B. Han, K. Qin, B. Yu et al., "Honeycomb-corrugation hybrid as a novel sandwich core for significantly enhanced compressive performance," Materials and Design, vol. 93, pp. 271-282, 2016.

[21] Y. Y. Lee, E. W. M. Lee, and C. F. Ng, "Sound absorption of a finite flexible micro-perforated panel backed by an air cavity," Journal of Sound and Vibration, vol. 287, no. 1-2, pp. 227-243, 2005.

[22] A. Sanada and N. Tanaka, "Extension of the frequency range of resonant sound absorbers using two-degree-of-freedom helmholtz-based resonators with a flexible panel," Applied Acoustics, vol. 74, no. 4, pp. 509-516, 2013.

[23] Y. Tang, S. Ren, H. Meng et al., "Hybrid acoustic metamaterial as super absorber for broadband low-frequency sound," Scientific Reports, vol. 7, p. 43340, 2017.

[24] B. Keshtegar, M. Bagheri, C. W. Fei, C. Lu, O. Taylan, and D. K. Thai, "Multi-extremum modified response basis model for nonlinear response prediction of dynamic turbine blisk," Engineering with Computers, 2021.

[25] D. Griese, J. D. Summers, and L. Thompson, "The effect of honeycomb core geometry on the sound transmission performance of sandwich panels," Journal of Vibration and Acoustics, vol. 137, no. 2, Article ID 021011, 2015.

[26] H. Meng, M. A. Galland, M. Ichchou, F. X. Xin, and T. J. Lu, "On the low frequency acoustic properties of novel multifunctional honeycomb sandwich panels with micro-perforated faceplates," Applied Acoustics, vol. 152, pp. 31-40, 2019.

[27] L. Han, C. Chen, T. Guo et al., "Probability-based service safety life prediction approach of raw and treated turbine blades regarding combined cycle fatigue," Aerospace Science and Technology, vol. 110, Article ID 106513, 2021.

[28] C. W. Fei, H. T. Liu, S. L. Li, H. Li, L. Q. An, and C. Lu, "Dynamic parametric modeling-based model updating strategy of aeroengine casings," Chinese Journal of Aeronautics, 2021.

[29] G. d. N. Almeida, E. F. Vergara, L. R. Barbosa, and R. Brum, "Low-frequency sound absorption of a metamaterial with symmetrical-coiled-up spaces," Applied Acoustics, vol. 172, p. 107593, Article ID 107593, 2021.

[30] F. Wu, W. Chen, and Z. Ju, "Research on low frequency broadband sound absorption metamaterial based on combined micro-perforated plate," Material China, vol. 40, no. 1, pp. 29-33, 2021.

[31] J. Guo, X. Zhang, Y. Fang, and Z. Jiang, "Wideband lowfrequency sound absorption by inhomogeneous multi-layer resonators with extended necks," Composite Structures, vol. 260, Article ID 113538, 2021.

[32] F. Bucciarelli and M. Meo, "Broadening sound absorption coefficient with hybrid resonances," Applied Acoustics, vol. 160, 2020 ISSN 0003-682X, Article ID 107136.

[33] J. Hou, H. Zhu, and S. Yuan Suwei, "Acoustic absorption characteristics of a flexible micro-perforated panel cavity with a flexible back one," Vibration and Shock, vol. 39, no. 15, pp. 156-162, 2020. 